\title{
Time-dependence of the electromechanical bending actuation observed in ionic-electroactive polymers
}

\author{
Patrick S. Bass, Lin Zhang and Z.-Y. Cheng* \\ Materials Research and Education Center, Auburn University, Auburn, AL 36849, USA \\ *chengzh@eng.auburn.edu
}

Received 27 January 2017; Revised 5 February 2017; Accepted 16 February 2017; Published 10 March 2017

\begin{abstract}
The characteristics of the electromechanical response observed in an ionic-electroactive polymer (i-EAP) are represented by the time $(t)$ dependence of its bending actuation $(y)$. The electromechanical response of a typical i-EAP — poly(ethylene oxide) (PEO) doped with lithium perchlorate (LP) — is studied. The shortcomings of all existing models describing the electromechanical response obtained in i-EAPs are discussed. A more reasonable model: $y=y_{\max } e^{-\tau / t}$ is introduced to characterize this time dependence for all i-EAPs. The advantages and correctness of this model are confirmed using results obtained in PEO-LP actuators with different LP contents and at different temperatures. The applicability and universality of this model are validated using the reported results obtained from two different i-EAPs: one is Flemion and the other is polypyrrole actuators.
\end{abstract}

Keywords: i-EAP; PEO; electromechanical response; model.

Electroactive polymers (EAPs), also named as artificial muscles, are a newly developed type of polymeric materials exhibiting giant electromechanical response. ${ }^{1,2}$ EAPs are lightweight, produce large actuation performance, show good fracture tolerance, and can be made into almost any shape. ${ }^{1}$ Therefore, a great deal of attention has been given to the EAPs over the past two decades for their ability to generate large electromechanical actuation under an applied electric field without the need for any moving parts, external motors or servos. ${ }^{2}$ This biomimetic functionality fosters rich possible applications in robotics, the medical field, microfluidics, aerospace applications, etc. ${ }^{2}$ The EAPs studied can be classified into two categories: electronic EAP (e-EAP) and ionic EAP (i-EAP). ${ }^{1}$

The electromechanical response observed in e-EAPs can originate from an electrostrictive effect, ${ }^{3}$ the Maxwell-stress (i.e., electrostatic force) effect, ${ }^{4,5}$ and the re-orientation of dipoles. ${ }^{6}$ The e-EAPs are usually good electrical insulators that can stand with a high electric field. They exhibit a fast electromechanical response with a linear strain and a response time from $\mu$ s to ns and their strain response is well defined by the electric field applied on it. Therefore, the electromechanical response of an e-EAP is usually characterized by the relationship between the electric induced strain and the electric field applied on. ${ }^{6,7}$ For the e-EAPs to exhibit a high strain, a high electric field $(>100 \mathrm{MV} / \mathrm{m})$ is usually required..$^{6,7}$

Regarding i-EAPs which include ionic polymer-metal composites (IPMCs),${ }^{8}$ conducting polymers, ${ }^{9}$ hydrogels, ${ }^{10}$ etc., when an electric field is applied on it, the ions in the polymer are forced to move so that the distribution of the ions changes with time, ${ }^{1,8-10}$ which leads to the accumulation of cations and anions onto different electrodes. The size disparity between the two types of mobile ions results in a differential expansion/shrinkage across the polymer matrix. ${ }^{1,8-11}$ Therefore, a bending actuation as the electromechanical response is induced in a single layer of an i-EAP film as shown in Fig. 1, where an i-EAP film without electric field is flat (i.e., straight in figure from the side view). The i-EAP film is bended to a circular curve when an electric field is applied on it. Therefore, i-EAPs can generate a giant displacement. The i-EAPs require very low electric fields, usually a few volts on an i-EAP film with a thickness from a few tens to hundreds $\mu \mathrm{m}$ even $\mathrm{mm}$. That is, the i-EAPs are closer to real muscle than e-EAPs so much more efforts have been spent on the i-EAPs than on the e-EAPs. Among the i-EAPs, IPMCs have received the largest amount of attention because they can achieve tip deflections/displacement in excess of $360^{\circ}$ while only requiring between 1 and $5 \mathrm{~V}$ DC to drive this response. ${ }^{1,2}$

Different with the e-EAPs, the i-EAPs exhibit a slow electromechanical response with a response time ranging from seconds to minutes. As shown in Fig. 2, the electromechanical response or actuation observed in an i-EAP is a highly nonlinear time dependence. Therefore, the characteristics of its electromechanical response are presented by the relationship between the actuation $(y)$ and time $(t)$ for an i-EAP under a constant voltage. The actuation of an i-EAP can be represented by either one of three parameters: strain in the film, the tip displacement, and tip-displacement angle $(\theta)$. It is well known that the actuation of an i-EAP under a constant voltage is monotonically increasing with time. All

This is an Open Access article published by World Scientific Publishing Company. It is distributed under the terms of the Creative Commons Attribution 4.0 (CC-BY) License. Further distribution of this work is permitted, provided the original work is properly cited. 


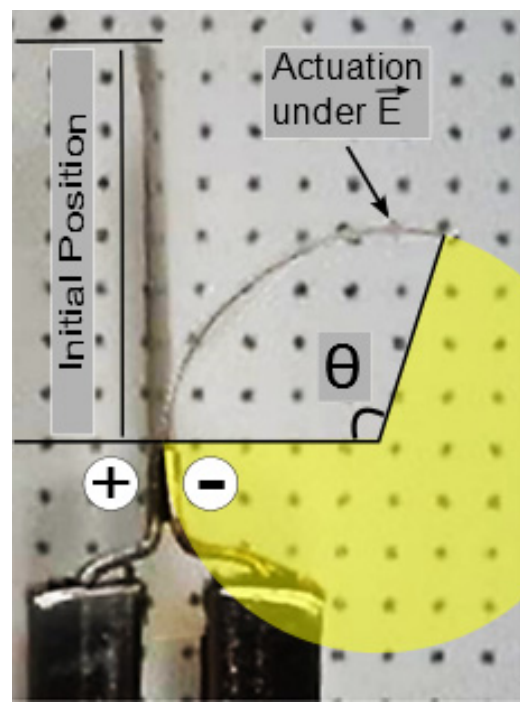

Fig. 1. The electromechanical response of i-EAP: initial position i-EAP film (side view) is flat without electric field; the electromechanical response of i-EAP under electric field is bending actuation. The actuation is characterized using tip-displacement angle, $\theta$. To calculate $\theta$ it is assumed that the overall length of i-EAP does not change with the electric field. Here the i-EAP actuator is PEO with 2.5 wt. $\%$ LP.

experimental results have shown that the electromechanical response or the actuation has three phases: at the beginning, the initial actuation is small and changes slow; then the actuation increases fast and large actuation is observed; eventually, the actuation is saturated with time. ${ }^{12}$ This threephased response can be described as slow, steady, and saturated. Accurately characterizing this behavior is important to deepen the understandings of i-EAPs and the key for incorporating these materials into commercial applications.

Various approaches have been used to develop equations/ models that can describe the time dependence of the actuation

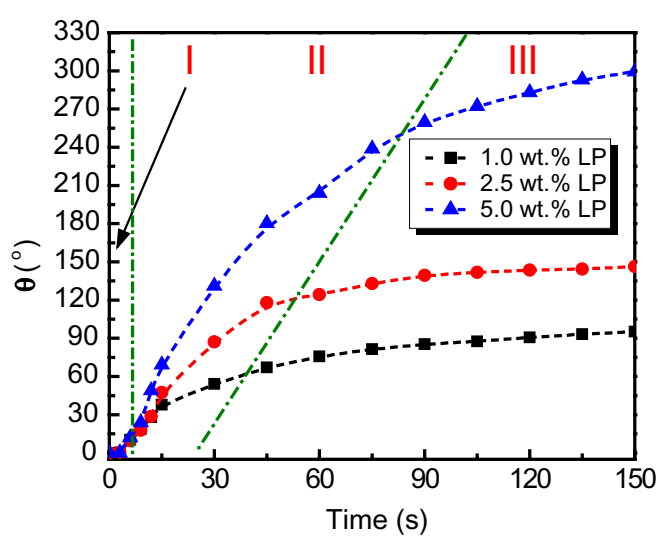

Fig. 2. Time dependence of the tip-displacement angle $(\theta)$ of PEOLP actuators at room temperature under $4 \mathrm{~V}$. The content of LP is indicated in figure. The time dependence of the tip-displacement angle shows three phase behavior. Three phases are labeled as I, II, and III. observed in an i-EAP under a constant voltage. The quantitative models describing the nonlinear actuation response, with varying degrees of success, have fallen into three categories: (1) physical models, where the underlying physical mechanisms, accounting for the various physical and chemical processes, are taken into consideration. ${ }^{13,14}$ Unfortunately, these processes are not very well understood and the resulting analysis has returned conflicting views of the root cause behind the bending. ${ }^{13,15}$ Additionally, equations generated from these models are typically difficult to correlate to experimental data and become impractical ${ }^{14,16}$; (2) black box models (i.e., empirical relationship) take the opposite approach and are introduced purely from experimental data with little consideration to the underlying mechanisms ${ }^{17}$; (3) gray box models act to bridge the two types of models wherein they combine well-known physical laws with empirically derived parameters. These models typically use finiteelement analysis ${ }^{18}$ or equivalent circuits ${ }^{19,20}$ to characterize the IPMC response. Some models require too many fitting constants so that they do not provide much information about the materials and are difficult to use.

Among the black box approaches, the equation introduced by Montazami et al., based on the normalized electromechanical strain of multilayer Nafion IPMCs, is the simplest with only two parameters as ${ }^{21}$ :

$$
y(t)=y_{\max }\left(1-e^{-t / \tau}\right) .
$$

Here, the strain inside the i-EAP film is originally used as the actuation variable. The two fitting constants are the maximum strain $\left(y_{\max }\right)$ and a time constant $\boldsymbol{\tau}$. Other equations have the similar form. That is, the actuation is dependent on the time through an exponential function, but multiple terms of exponential functions are used. For example, in an equation induced by Kanno et al., the tipdisplacement is described using four exponential functions with four different time constants, which results in eight fitting constants. ${ }^{17}$

For the gray box approach, Jain et al. used an equivalentcircuit approach to simulate the IPMC and resulted in the tipdisplacement angle as a function of time as ${ }^{19}$ :

$$
y(t)=\frac{K}{b-a} e^{-a t}+\frac{K}{a-b} e^{-b t},
$$

where three fitting constants $(K, a>0$, and $b>0)$ are used. Equation (2) has some similarities with Eq. (1). One may consider Eq. (2) as an expansion of Eq. (1) by having two different time constants. Shi et al. also used an equivalent circuit diagram to describe a Nafion-based IPMC, incorporating two additional resistors to account for the gold electrodes. ${ }^{20}$ Their work led to the tip-displacement being described as:

$$
y(t)=Q\left(\frac{1-b}{a} e^{-a t}+b t+\frac{b-1}{a}\right),
$$

where three fitting constants $(Q, a, b)$ are used. One may treat Eq. (3) as a modification of Eq. (1) by adding a linear term. 
In this work, a typical IPMC - poly(ethylene oxide) (PEO) doped with lithium perchlorate (LP) - is studied. PEO is also a well-known solid-state electrolyte, ${ }^{22}$ capable of having both cations and anions diffuse through its matrix ${ }^{23,24}$ under an applied electric field. As a typical IMPC, PEO has been well studied ${ }^{25}$ and various approaches have been employed to improve the electromechanical response of the PEO and in combination with other polymer systems. ${ }^{12,25,26}$ PEO-LP has a response time in tens of seconds, which provides an opportunity to have an accurate measurement on the time dependence of the actuation.

PEO $\left(M_{v}=100,000\right)$ and LP were obtained from Sigma Aldrich. Solutions comprised PEO with LP being dissolved into deionized water. In this study, samples with different LP contents $(1.0,2.5$, and $5.0 \mathrm{wt} . \%)$ were prepared using a solution casting process, in which the solution was cast on to glass substrate at $65^{\circ} \mathrm{C}$ for $90 \mathrm{~min}$. They were then rapidly cooled and peeled. Gold was sputtered onto both surfaces $(\sim 20 \mathrm{~nm})$ using a Pelco SC-6 sputter coater to act as electrodes. The thickness of free-standing films was measured by using a Mitutoyo 543-252 Absolute Digimatic Indicator. The average thickness of the samples is $55 \pm 5 \mu \mathrm{m}$. The samples used for the electromechanical response study had a size of $19.1 \mathrm{~mm} \times 6.35 \mathrm{~mm}$. For each composition, three samples were studied to confirm the results were repeatable. For each sample, the actuation was measured twice to show the electromechanical response is reversible. To characterize the time dependence of the actuation for these i-EAPs, a video tape of the actuation was made for the sample under a constant voltage. The compiled images shown in Fig. 1 are taken from video screenshots: the one on left is the i-EAP without electric field, the one on the right is the sample under an electric voltage of $4 \mathrm{~V} \mathrm{DC}$, with corresponding electric field of $0.07 \mathrm{~V} /$ $\mu \mathrm{m}$ or $70 \mathrm{~V} / \mathrm{mm}$. In this study, the tip-displacement angle, $\theta$, is used as the actuation response. That is, for a sample under a constant voltage, $\theta$ at different times was calculated using the still images taken from video and used as the actuation.

Experimental results of the prepared i-EAPs at room temperature are shown in Fig. 2, where a voltage of $4 \mathrm{~V} \mathrm{DC}$ was used for all the samples. Clearly, the three actuation phases of slow, steady, and saturated are observed and labeled as I, II, and III, respectively, in the figure. However, the time period for each phase is different. These are consistent with the results reported for other i-EAPs. ${ }^{12}$ The higher the LP concentration, the longer is the phase II process. The results shown in Fig. 2 indicate that the response time increases with increasing LP content.

The experimental results shown in Fig. 2 are fitted using Eqs. (1)-(3). It is found that all three models can fit the experimental results as shown in Fig. 3(a), where the sample with $2.5 \mathrm{wt} . \% \mathrm{LP}$ is presented as an example. However, it is found that all three models cannot fit the results well at the beginning (i.e., phase I process and earlier phase II process). Clearly, all the fitting results are significantly higher than the experimental results in this time period.

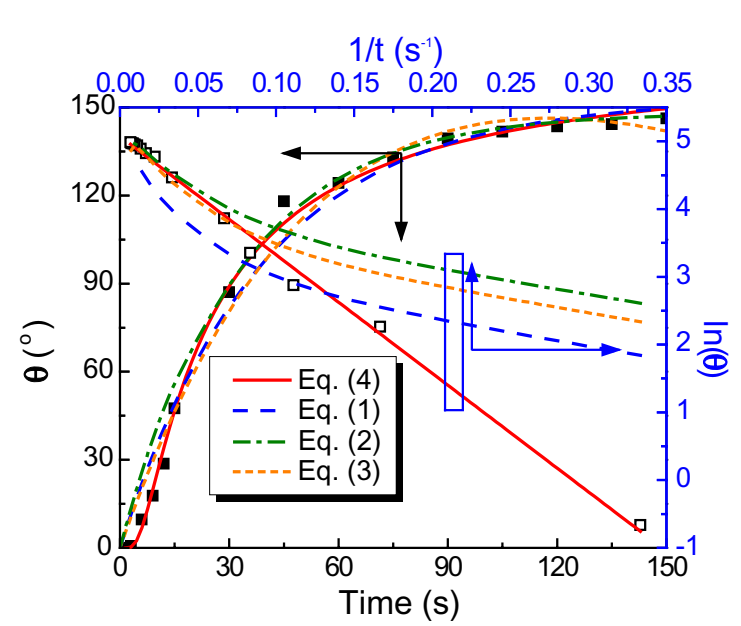

(a)

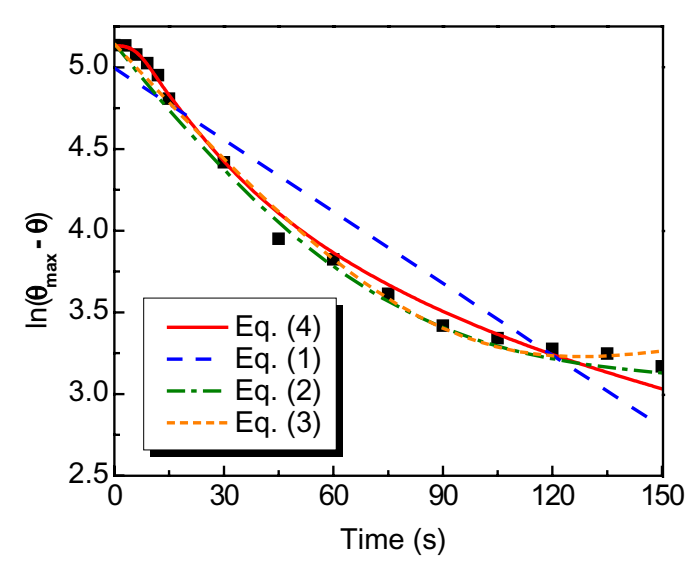

(b)

Fig. 3. (a) Time dependence of the tip-displacement angle of PEO with 2.5 wt.\% LP under $4 \mathrm{~V}$ at room temperature with the fitting curves using different equations and the plot of $\log (\theta)$ versus $1 / t$ with the fitting curves. (b) The plot of $\ln \left(y_{\max }-y\right)$ versus $t$.

To further analyze the results, the fitting constants for each equation are presented in Table 1. By physics, it is expected that the change in the fitting constants should somehow reflect the change in the LP content and the sign of every fitting constant should be the same for all i-EAPs. In other words, as the LP content increases, a fitting constant should either decrease or increase, but would not change its sign. For Eq. (3), it is found that $Q$ increased with LP content, while $a$ and $b$ both decreased. However, the sign of fitting constant $b$ changes from positive for samples with 1.0 and $2.5 \mathrm{wt} . \% \mathrm{LP}$ to negative for sample with $5.0 \mathrm{wt} . \% \mathrm{LP}$, which is against the physics of i-EAPs. For Eq. (2), it is found that the value of $b$ increases and, then, decreases with increasing LP content, which is against the physics of i-EAP. It is also found that the increase ( $\sim 1.2$ times) in the value of $a$ from samples with 2.5 wt. \% LP to $5.0 \mathrm{wt} . \% \mathrm{LP}$ is three orders lower than that $\left(\sim 1.2 \times 10^{3}\right.$ times $)$ from samples with $1.0 \mathrm{wt} . \% \mathrm{LP}$ to 2.5 wt.\% LP, which is unreasonable considering the change in LP 
Table 1. Fitting constants used in Eqs. (1)-(4) to fit the results shown in Fig. 2.

\begin{tabular}{|c|c|c|c|c|c|c|c|c|c|c|}
\hline \multirow[b]{2}{*}{ LP content (wt.\%) } & \multicolumn{2}{|c|}{ Eq. (4) } & \multicolumn{2}{|c|}{ Eq. (1) } & \multicolumn{3}{|c|}{ Eq. (2) } & \multicolumn{3}{|c|}{ Eq. (3) } \\
\hline & $Y_{\max }$ & $\tau(\mathrm{s})$ & $Y_{\max }$ & $\tau(\mathrm{s})$ & $K(\% / \mathrm{s})$ & $a(1 / \mathrm{s})$ & $b(1 / \mathrm{s})$ & $Q(\%)$ & $a(1 / \mathrm{s})$ & $b\left(\times 10^{-3}\right)$ \\
\hline 1.0 & $104^{\circ}$ & 14.8 & $92^{\circ}$ & 55.7 & 2.25 & $8.80 \times 10^{-6}$ & 0.023 & 2.9 & 0.036 & 1.50 \\
\hline 2.5 & $169^{\circ}$ & 19.7 & $147^{\circ}$ & 68.5 & 4.75 & $1.09 \times 10^{-5}$ & 0.032 & 4.5 & 0.031 & 0.16 \\
\hline 5.0 & $345^{\circ}$ & 22.8 & $339^{\circ}$ & 66.0 & 5.25 & $1.27 \times 10^{-2}$ & 0.0017 & 5.0 & 0.011 & -2.20 \\
\hline
\end{tabular}

content. Additionally, the fitting results from Eq. (3) show some reduction in the actuation with time. That is, after $120 \mathrm{~s}$, the fitting response decreases with time, which is against the experimental results and the physics of i-EAPs. Actually, both Eqs. (2) and (3) show something contradictory to the physics of i-EAPs. For Eq. (2), one can get that the actuation initially increases from zero with time and, then, decreases with time and eventually reaches to zero, which is certainly against the experimental results and physics of i-EAPs. Equation (3) indicates that the actuation initially increases from zero with time and, then, decreases slightly with time and, finally, increases with time. The final stage from the linear term of Eq. (3) results in an infinite actuation, which is again unreasonable by the physics. Therefore, one may conclude that both Eqs. (2) and (3) are invalid to characterize the time dependence of the actuation observed in i-EAPs.

Regarding Eq. (1), the fitting constant shows some irregular change. For example, when the LP content is changed from 1.0 to $2.5 \mathrm{wt} . \%$, the time constant increases from 55.7 to $68.5 \mathrm{~s}$. However, when the LP content is changed from 2.5 to $5.0 \mathrm{wt} . \%$, the time constant decreases from 68.5 to $66.0 \mathrm{~s}$. That is, the fitting time constant shows some irregular change with LP content, which is unreasonable by the physics. Therefore, a new relationship/model is needed to characterize the time dependence of the actuation observed in i-EAPs. Considering the fact that all current models cannot fit the actuation in the phase I process, a new relationship/model is introduced as:

$$
y=y_{\max } e^{-\tau / t},
$$

where $\tau$ and $y_{\max }$ are the time constant and maximum actuation. Here the tip-displacement angle $(\theta)$ is used as the measure of the actuation. From a physics point of view, Eq. (4) follows the expectation: (1) as the time approaches to zero, the actuation is zero; (2) the actuation monotonically increases with time; (3) as the time approaches very long, the actuation reaches its maximum $\left(y_{\max }\right)$; (4) this relationship reflects the three phase behavior in the actuation. Similar to Eq. (1), there are only two fitting constants.

The results shown in Fig. 2 are fitted using Eq. (4). The fitting constants are presented in Table 1 and the fitting curve is presented in Fig. 3(a) as an example. From Fig. 3(a), one can find that the fitting results using Eq. (4) is better than the other three. First of all, the fitting results of Eq. (4) reflect the actuation during phase I process very well. Additionally, the fitting constants shown in Table 1 indicate that the fitting constants of Eq. (4) coordinately change with the LP content. For example, the time constant increases with the LP content, which is consistent with the results shown in Fig. 2.

To further show the advantage of Eq. (4) over others, the fitting results using both Eqs. (1) and (4) are further compared. Equation (4) can be written as

$$
\ln y=\ln y_{\max }-\tau / t .
$$

That is, the new model would result in a linear relationship between the logarithm of actuation, $\ln y$, and the reciprocal of time, $1 / t$. The plot of $\ln y$ versus $1 / t$ is shown in Fig. 3(a). Clearly, the experimental results show a very good linear relationship. However, the tendency of Eq. (1) is completely different with the experimental results, especially at large $1 / t$ (i.e., the phase I process).

Equation (1) can be written as:

$$
\ln \left(y_{\max }-y\right)=\ln y_{\max }-t / \tau .
$$

That is, there would be a linear relationship between the $\ln \left(y_{\max }-y\right)$ and the time. The experimental results shown in Fig. 2 are plotted as $\ln \left(y_{\max }-y\right)$ versus $t$. As shown in Fig. 3(b), the fitting results of Eq. (6) do not represent the tendency of the experimental results at all.

The results shown in Fig. 3 and Table 1 clearly demonstrate the advantage and correctness of Eq. (4) over other models. To further confirm it, both Eqs. (1) and (4) are used to analyze the electromechanical response of the samples with $2.5 \mathrm{wt} . \% \mathrm{LP}$ at different temperatures $\left(10^{\circ} \mathrm{C}, 20^{\circ} \mathrm{C}\right.$, $30^{\circ} \mathrm{C}, 40^{\circ} \mathrm{C}, 50^{\circ} \mathrm{C}$ ). Both equations can fit the results obtained at each temperature. However, the fitting using Eq. (1) cannot reflect the electromechanical response at the beginning (i.e., the phase I process and earlier phase II process), which is exactly what was observed above in Fig. 3. Regarding fitting constants using Eq. (4), it is concluded that the saturated actuation $\left(y_{\max }\right)$ increases with increasing temperature and that the time constant $(\boldsymbol{\tau})$ decreases with increasing temperature. A higher temperature means that the ions in i-EAPs have greater mobility, which would result in a fast response or a smaller time constant. For the polymer matrix, the higher is the temperature, the smaller is its elastic modulus. Therefore, for the same ion accumulation in the PEO, a larger actuation would be obtained at a higher temperature. In other words, the fitting results using Eq. (4) are consistent with the physics of i-EAPs. 
To further validate applicability and universality of Eq. (4), the experimental data reported for two other i-EAPs are analyzed: one is Flemion and the other is polypyrrole (PPy) actuator. ${ }^{27,28}$ Flemion is a commercial IPMC and has a response time of $10^{2} \mathrm{~s}^{27} \mathrm{PPy}$ is a conducting polymer, representing a different type of i-EAPs, and has a much fast response (i.e., a shorter response time). ${ }^{28}$

The results shown in Fig. 4(a) are the electromechanical response of a Flemion actuator under $3 \mathrm{~V} \mathrm{DC},{ }^{27}$ while the results shown in Fig. 4(b) are obtained from a PPy actuator under $0.75 \mathrm{~V} \mathrm{DC}{ }^{28}$ The experimental data shown for the Flemion films was acquired from the still images published in Ref. 27 while the PPy data was acquired from a timedependent figure that displayed changes in tip-displacement angle in Ref. 28. Both Eqs. (1) and (4) are used to fit the

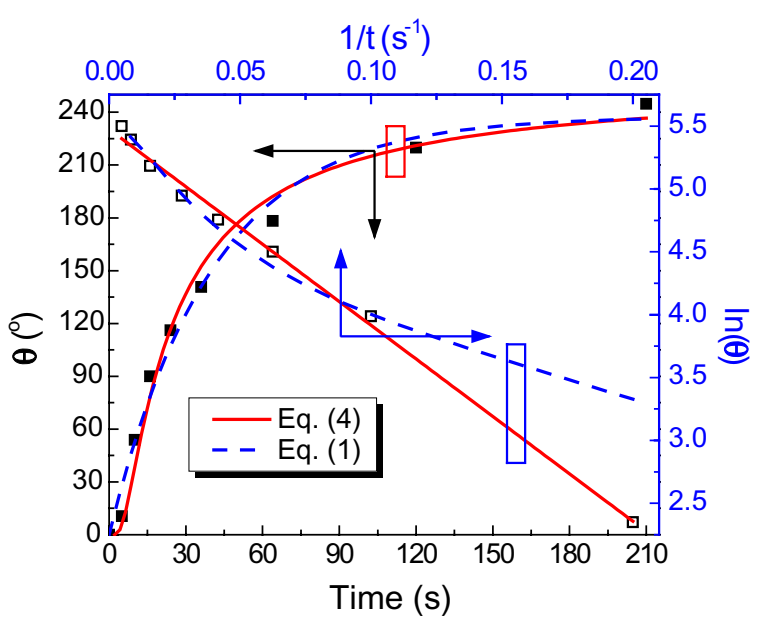

(a)

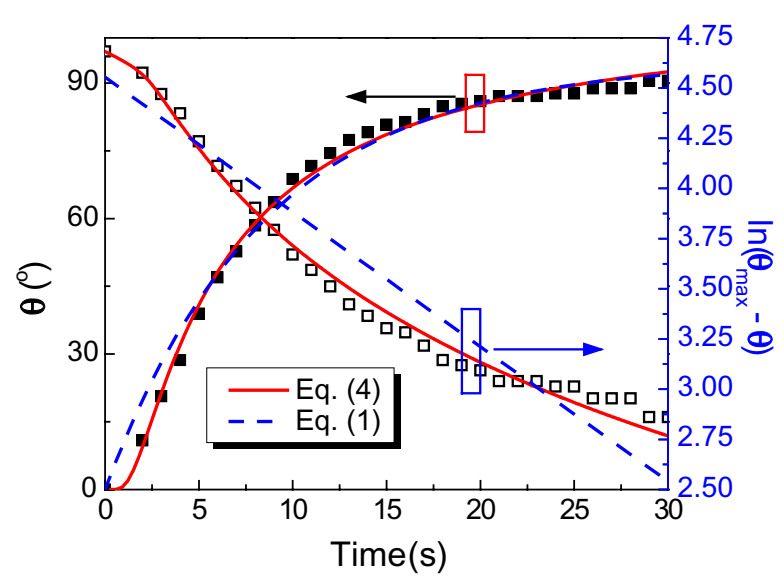

(b)

Fig. 4. (a) Time dependence of the tip-displacement angle of Flemion, tested at $3 \mathrm{~V}$, with fitting curves using Eqs. (1) and (4) and the plot of the $\log (\theta)$ versus $1 / t$. (b) Time dependence of the tipdisplacement angle of polypyrrole, tested at $0.75 \mathrm{~V}$, with fitting curves using Eqs. (1) and (4) and the plot of $\ln \left(y_{\max }-y\right)$ versus $t$. The experimental data in (a) is from Ref. 27 and the data in (b) is from Ref. 28. experimental results. Again both equations can fit the experimental results, but the fitting results using Eq. (1) do not reflect the actuation behavior at the beginning. Additionally, both Eqs. (5) and (6) are also used to check the tendency of the electromechanical response. In Fig. 4(a), the plot of $\ln (y)$ versus $1 / t$ is presented, while the plot of $\ln \left(y_{\max }-y\right)$ versus $t$ is presented in Fig. 4(b). Again the experimental results show that Eq. (5) can well describe the electromechanical response of i-EAPs. These results again show the advantage of Eq. (4) over Eq. (1).

It is experimentally found that the electromechanical response of an i-EAP under a constant voltage has three phases: phase I process - slow; phase II process - steady increase; phase III process - saturation. Current models used to describe this electromechanical response are studied using the experimental results obtained in PEO doped with LP with different contents. The shortcomings of all existing models for fitting the experimental results are discussed. Some are physically incorrect. All cannot reflect the electromechanical response at the beginning (i.e., the phase I and earlier phase II process). Additionally, the fitting constants are inconsistent with the physics. A new and reasonable model, Eq. (4), is introduced to characterize the electromechanical response of i-EAPs. The new relationship also provide an easy way to fit the results: a linear relationship is obtained by plotting the logarithm of the actuation versus the reciprocal of time. The correctness and advantages of the new model are also confirmed using the electromechanical response of the PEO-LP actuators at different temperatures. The applicability and universality of the new model are validated using the reported results from other i-EAPs: one is Flemion and the other is PPy.

\section{Acknowledgments}

P.B. has been financially supported by the National Science Foundation through the Auburn University IGERT (Award \#: 1069004). This work was partially funded by NSF-IGERT and USDA-NIFA (Grant \#: 2014-60722-21694).

\section{References}

${ }^{1}$ Y. Bar-Cohen and Q. Zhang, MRS Bull. 33(3), 173 (2008).

${ }^{2}$ Y. Bar-Cohen, Electroactive Polymer (EAP) Actuators as Artificial Musles: Reality, Potential, and Challenges (SPIE Press, Bellingham, 2004).

${ }^{3}$ Z.-Y. Cheng, V. Bharti, T. B. Zu, H. S. Xu, T. Mai and Q. Zhang, Sensors and Actuators A: Physical 90(1), 138 (2001).

${ }^{4}$ R. Pelrine, R. Kornbluh, Q. Pei and J. Joseph, Science 287(5454), 836 (2000).

${ }^{5}$ Q. Zhang, H. Li, M. Poh, F. Xia, Z.-Y. Cheng, H. Xu and C. Huang, Nature 419(6904), 284 (2002).

${ }^{6}$ Z. Cheng and Q. Zhang, MRS Bull. 33(3), 183 (2008).

${ }^{7}$ Z. Li, Y. Wang and Z.-Y. Cheng, Appl. Phys. Lett. 88(6), 062904 (2006). 
${ }^{8}$ M. Shahinpoor, Electrochim. Acta 48(14), 2343 (2003).

${ }^{9}$ E. W. Jager, E. Smela and O. Inganäs, Science 290(5496), 1540 (2000).

${ }^{10}$ F. Carpi and E. Smela, Biomedical Applications of Electroactive Polymer Actuators (John Wiley \& Sons, 2009).

${ }^{11}$ J. D. Madden, N. A. Vandesteeg, P. A. Anquetil, P. G. Madden, A. Takshi, R. Z. Pytel, S. R. Lafontaine, P. A. Wieringa and I. W. Hunter, IEEE J. Ocean. Eng. 29(3), 706 (2004).

${ }^{12}$ S. K. Mahadeva, S. Yun and J. Kim, J. Intell. Mater. Syst. Struct. 20(10), 1141 (2009).

${ }^{13}$ S. Nemat-Nasser and J. Y. Li, J. Appl. Phys. 87(7), 3321 (2000).

${ }^{14}$ Y. Cha, M. Aureli and M. Porfiri, J. Appl. Phys. 111(12), 124901 (2012).

${ }^{15} \mathrm{~S}$. Tadokoro, S. Yamagami, T. Takamori and K. Oguro, SPIE's 7th Annual Int. Symp. Smart Structures and Materials, Vol. 3987(1) (2000), p. 92.

${ }^{16}$ D. Pugal, K. J. Kim and A. Aabloo, J. Appl. Phys. 110(8), 084904 (2011).

${ }^{17}$ R. Kanno, A. Kurata, M. Hattori, S. Tadokoro, T. Takamori and K. Oguro, in Proc. Japan-USA Symp. on Flexible Automation, Vol. 2 (1) (1994), p. 691.
${ }^{18}$ W. Yim, J. Lee and K. J. Kim, Bioinspir. Biomim. 2(2), S31 (2007).

${ }^{19}$ P. Jain, S. Datta, S. Majumder and A. Dutta, Int. J. Adv. Robot. Syst. 8(1), 1 (2011).

${ }^{20}$ L. Shi, S. Guo, M. Li, S. Mao, N. Xiao, B. Gao, Z. Song and K. Asaka, Sensors 12(12), 16732 (2012).

${ }^{21}$ R. Montazami, S. Liu, Y. Liu, D. Wang, Q. Zhang and J. R. Heflin, J. Appl. Phys. 109(10), 104301 (2011).

${ }^{22}$ J. W. Fergus, J. Power Sources 195(15), 4554 (2010).

${ }^{23}$ Z. Gadjourova, Y. G. Andreev, D. P. Tunstall and P. G. Bruce, Nature 412(6846), 520 (2001).

${ }^{24}$ K. Hayamizu, E. Akiba, T. Bando, Y. Aihara and W. S. Price, Macromolecules 36(8), 2785 (2003).

${ }^{25}$ M. Shahinpoor and K. J. Kim, Appl. Phys. Lett. 80(18), 3445 (2002).

${ }^{26}$ C. Plesse, A. Khaldi, Q. Wang, E. Cattan, D. Teyssié, C. Chevrot and F. Vidal, Smart Mater. Struct. 20(12), 124002 (2011).

${ }^{27}$ S. Nemat-Nasser and Y. Wu, J. Appl. Phys. 93(9), 5255 (2003).

${ }^{28}$ R. Mutlu, G. Alici, X. Xiang and W. Li, Mechatronics 24(3), 241 (2014). 